\title{
The Sensitivity of a Water Distribution System to Regional State Parameter Variations
}

\author{
Philip R. Page (iD) \\ Built Environment, Council for Scientific and Industrial Research (CSIR), Pretoria 0184, South Africa \\ Correspondence should be addressed to Philip R. Page; pagepr7@gmail.com
}

Received 4 August 2017; Revised 6 April 2018; Accepted 23 April 2018; Published 29 May 2018

Academic Editor: Suzanne M. Shontz

Copyright (C) 2018 Philip R. Page. This is an open access article distributed under the Creative Commons Attribution License, which permits unrestricted use, distribution, and reproduction in any medium, provided the original work is properly cited.

\begin{abstract}
The sensitivity of a pressurised water distribution system (WDS) to state parameter variations is studied. A novel local regional sensitivity analysis (LRSA) approach is introduced which applies the same change to a collection of parameters, called a region. For example, sensitivity to suburbs can be studied. General analytical (using algebraic methods) results are derived. They show how sensible conclusions arise from LRSA and state this dependence of the WDS on regions for the first time. For most cases, the WDS is 1.852-2 times more sensitive to pipe roughness coefficients than to pipe lengths. In most cases, when certain pipes do not have minor losses, the WDS is $4.871-5.333$ times more sensitive to pipe diameters than to pipe lengths. Hence, the WDS is the most sensitive to pipe diameters, medium sensitive to pipe roughness coefficients, and least sensitive to pipe lengths. For most cases, when all reservoir and tank elevations (and heads) remain the same, changes of other elevations do not change flow rates and change the pressures in a simple additive way. In most cases, when all the reservoir water surface elevations are changed together, the flow rates remain unchanged, and the pressures change in a simple additive way.
\end{abstract}

\section{Introduction}

The study of sensitivity analysis [1, 2], employed here, is concerned with the following:

(1) Forward use: how much the outputs depend on each or some of the inputs.

(2) Inverse use: how uncertainty in the outputs can be apportioned to different sources of uncertainty in the inputs.

Sensitivity analysis may be performed for a variety of reasons, including exploration of model response to specific inputs (a forward use) or identification of key model inputs (an inverse use). The former can be useful for verification or validation of the model [3] and the latter for the calibration of the model [4, Section 14.7].

A pressurised water distribution system (WDS) depends on various state parameters (i.e., "inputs", e.g., pipe lengths) that are characteristics of the infrastructure and water status [5-7]. Numerical models then predict the water hydraulic variables (i.e., "outputs", e.g., flow rates and pressures) from the state parameters (water quality properties are not considered here). For the objective of a particular sensitivity study, there is typically interest in a particular hydraulic water property, or a combination of such properties, called the indicator (or index) function [8-13]. For example, the minimum pressure in the system [14, p. 131], or the average pressure in the entire system, might be of interest. The sensitivity analysis answers the question: What is the sensitivity of an indicator function to change in the parameters that it depends on?

Various sensitivity analysis methods exist: some are more mathematical and others more statistical, in nature $[1,3]$. Statistical methods typically approach the subject by assuming that parameters are distributed according to a probability density function. There are numerous applications to the WDS, as recently reviewed [15]. Common methods used in WDS applications include Monte Carlo $[16,17]$ and an accompanying approximation called first-order second moment (FOSM) [8, 16], which uses the sensitivity-matrix evaluated at the mean of the distribution. Fuzzy sets, as recently reviewed [18], have also been used for WDS applications.

The following mathematical methods exist: nominal range sensitivity analysis (NRSA) [3] (also known as "local sensitivity analysis", "threshold analysis", "one-at-a-time", or 
"one-factor-at-a-time") and sensitivity-matrix methods [1, 17]. NRSA and sensitivity-matrix methods are applicable to deterministic models [2], such as the hydraulic pipe network models used for a WDS, considered here. These methods, respectively, apply to changes of single and multiple state parameters. NRSA evaluates the effect on model outputs exerted by individually varying only one of the model inputs across its entire range of plausible values, while holding all other inputs at their nominal or base-case values (i.e., fix them locally) [2]. NRSA can be repeated for any number of individual model inputs. An extension to multiple parameters, with a linearization approximation, is the sensitivity-matrix, which consists of first-order partial derivatives of outputs with respect to (w.r.t.) inputs (Jacobian matrices) $[1,3]$. Local sensitivity analysis methods perturb some inputs at a time, while the remaining inputs are fixed to base-case values. NRSA and sensitivity-matrix methods are local methods [3].

Recent applications of mathematical sensitivity analysis to the hydraulics of a WDS include a GIS-based sensitivity analysis method based on NRSA used in several analyses of an Austrian-based group. For the effect of continuous change of parameters on hydraulic variables, of interest here, this method was applied to calibration, pipe diameter design, and input uncertainty assessment [10], and vulnerability identification [11]. Other recent applications include the sensitivitymatrix method for ranking the relative importance of pipes [9], leakage localisation [19], and studying demands [20].

Since NRSA is the easiest sensitivity analysis method to implement and understand [3], a novel sensitivity analysis formalism that extends this method is developed, called local regional sensitivity analysis (LRSA). Like the NRSA and sensitivity-matrix methods, it is local and should hence preferably be applied to problems where this is an advantage, or not a disadvantage. Examples of such problems are model verification or validation [3].

In NRSA and sensitivity-matrix methods different changes are applied to each parameter individually. LRSA extends these methods to allow the same change to apply to a collection of parameters, called a region. The importance of the study of parameter regions has been emphasized [16]. For example, by breaking the entire system into suburbs, a complicated calibration problem can be studied incrementally [4, Section 14.8].

The novelty of LRSA is that it allows, in an easy way, consideration of the sensitivity of the WDS to state parameter regions, not just individual state parameters. The unique contributions that are made to the study of a WDS are as follows:

(i) Ease of understanding: a complicated WDS is easier to understand if the number of parameters it is sensitive to is reduced. This is done by lumping parameters together in regions.

(ii) Simplicity of analysis: because the parameters in the WDS can be grouped into regions, the process of sensitivity analysis is simplified.

(iii) Dependence on the collective (the region): because a change of the collective is made, the sensitivity of the WDS to the collective can be studied.
Numerical sensitivity calculations have been performed for two networks [21], using the LRSA techniques in Section 3.2. In this paper, several results are derived, which are of a general nature. The results explicitly state the dependence of a WDS on state parameter regions for the first time. The results also serve as consistency checks with the current body of knowledge to show how sensible results arise from LRSA. One motivation for using these results as consistency checks is that they do not depend on a linearization approximation.

The results apply to the standard NRSA and sensitivitymatrix methods as special cases. Hence the results do not require adoption of the LRSA method and stand in their own right. For the NRSA and sensitivity-matrix methods, the results have not been found to be explicitly stated elsewhere.

The paper is organized as follows. LRSA is introduced in Section 2 and then developed in Section 3 for a single state parameter region. The single region results are introduced in Section 4; the pipe parameter scaling laws are stated in Section 5 and the node elevation uncertainty results in Section 6. The multiple state parameter region results are introduced in Section 7, with the generalized pipe parameter scaling laws and node elevation uncertainty results in Sections 8 and 9 , respectively. The types of sensitivity analysis covered by LRSA, and how these relate to conventional methods, are described in Section 10; and Section 11 summarizes the conclusions. The Appendix contains single region proofs of results and illustrative examples.

\section{Introduction to Local Regional Sensitivity Analysis}

A central characteristic of the sensitivity analysis developed below is that the same change is made for all the state parameters in a single set of parameters (either $\mathscr{X}$ or $\mathscr{Z}$ ), as is partially implemented in some current network models for demand [4, Section 14.7], [5]. The effect of the change on a single indicator function (composed of all the water flow rates and pressures in a single set of links $\mathscr{L}^{\oplus}$ and nodes $\mathscr{M}^{\oplus}$ ) is then determined mathematically (generalization beyond a "single" set of parameters will be made later in the paper but only serves to complicate matters at this stage). There is hence an association

$$
\begin{gathered}
\text { State parameter region, either } \mathscr{X} \text { or } \mathscr{Z} \\
\longleftrightarrow \text { Variable region }\left(\mathscr{L}^{\oplus}, \mathscr{M}^{\oplus}\right) .
\end{gathered}
$$

The two regions are in general unrelated, and a region does not have to be spatially connected. However, in practical problems $\mathscr{X}$ or $\mathscr{Z}$ often represents a connected region in space and similarly for $\left(\mathscr{L}^{\oplus}, \mathscr{M}^{\oplus}\right)$. For example, $\mathscr{X}$ or $\mathscr{Z}$ can represent a suburb inside a WDS, while $\left(\mathscr{L}^{\oplus}, \mathscr{M}^{\oplus}\right)$ can represent the end-nodes of the entire system, where pressure is often the lowest.

The sensitivity analysis considers two types of continuous state parameter change.

Proportional Change $\left(X_{i} \rightarrow r X_{i}\right.$ for all $\left.i \in \mathscr{X}\right)$. These are appropriate for parameters which can only have one sign, 
e.g., pipe length. There are three important characteristics of proportional change:

(1) $r$ is dimensionless, so change made to different types of parameters can be compared.

(2) The change emphasizes the larger parameter values. For example, if a set of pipe lengths is changed by the same amount $r$, the pipes with the larger lengths are changed more. This is sensible, since the larger length pipes should be more "important" when change due to pipe lengths is considered.

(3) The change to the individual parameters can naturally be interpreted as a single property of the collective. For example, if a set of pipe lengths in a connected region is changed by the same amount $r$, the total pipe length of the region is changed by $r$. This means that the collective pipe length of the region is changed in a sensible way.

Additive Change $\left(Z_{j} \rightarrow Z_{j}+z\right.$ for all $\left.j \in \mathscr{Z}\right)$. These are appropriate for parameters which can have different signs, e.g., elevation. There are two important characteristics of additive change:

(1) The change to the parameters is independent of the parameter values. For example, elevations are changed by the same amount $z$. This is sensible, since the absolute size of an elevation is arbitrary, because all elevations refer to a reference elevation (e.g., mean sea level $[5,14])$.

(2) The change to the individual parameters can naturally be interpreted as a single property of the collective. For example, if a set of elevations in a connected region is changed by the same amount $z$, the elevation of the entire region is changed by $z$. This means that the collective elevation of the region is changed in a sensible way.

All the parameters in a state parameter region are changed together, so that the changes of the parameters are correlated in a specific way. It is proposed that it is sufficient for a parameter region to be changed according to only one of two types of changes: proportional or additive.

\section{Sensitivity Analysis Formulation: Single Region}

3.1. Parameter Variation: Proportional Change. Standard modelling of a WDS requires the construction of a network with internal nodes, and links (or lines) joining the external and internal nodes [5-7] (Figure 1). Water flows at rate $q_{l}$ through a link, and every internal node has pressure head $p_{m}$ at the position of the node. Pressure is defined in such a way that atmospheric pressure is zero. The variables

$$
\begin{array}{cl}
q_{l} & l \in \mathscr{L}^{\odot}, \\
p_{m} & m \in \mathscr{M}^{\odot}
\end{array}
$$

are obtained as a solution of a numerical model. Here $\mathscr{L}^{\odot}$ denotes all links and $\mathscr{M}^{\odot}$ all internal nodes. By convention,

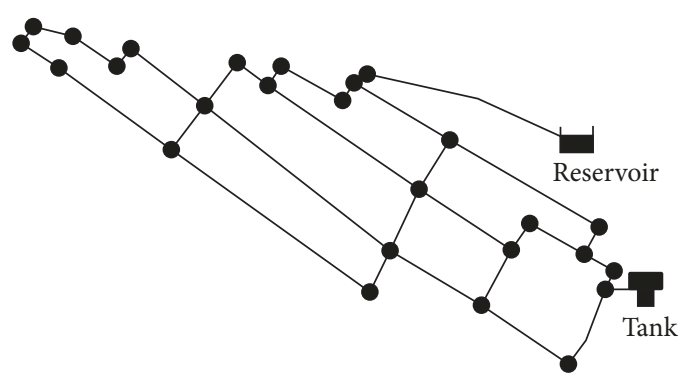

FIGURE 1: WDS with two external nodes (a reservoir and tank). The internal nodes are indicated by circles.

flow rates are positive for the base-case solution, and a pressure is usually positive (when a flow rate is negative, reversing the direction of the corresponding link will make it positive). Reservoirs or tanks are by definition external nodes.

The extended-period numerical model contemplated uses standard concepts and terminology [7], as represented by the industry-standard public domain software EPANET 2 [5]. To solve the equations, the state of the system must first be specified by a set of base-case parameters $X_{i}$ and $Z_{j}$ $[5-7,22,23]$. The following types of parameters fall in each category:

Proportional Change Parameters $X_{i}$.

(i) Pipe lengths $L$

(ii) Pipe diameters $D$

(iii) Pipe roughness coefficients $C$ (Hazen-Williams), $N$ (Chezy-Manning) or for Darcy-Weisbach

(iv) Minor loss coefficients $K$

(v) Water demand $d$, which usually has the same sign at a specific node (e.g., for customer water supply, $d$ is positive; for a borehole $d$ is negative)

(vi) Pump curve, valve, and emitter (sprinkler) parameters [5]

(vii) Tank diameter $D_{T}$ (tank shape parameters)

Additive Change Parameters $Z_{j}$.

(i) Elevations

(ii) Reservoir piezometric head (equal to the water surface elevation if the reservoir is under atmospheric pressure) [5]

(iii) Tank: the water surface elevation (this an important parameter relevant to hydraulic analysis), bottom and top water surface elevations of the tank.

The formalism also covers continuous parameters which specify curves (e.g., parameters that specify a pump curve, valve head-loss curve, or tank shape [5]).

The solutions of the equations can formally be denoted as a nonlinear function $f$, such that the solution vector $(\mathbf{q}, \mathbf{p})=$ $f(\mathbf{X}, \mathbf{Z})$. Inverse sensitivity analysis involves the inversion of $f$, which is usually complicated. However, a meaningful case where inversion is simple is developed in the formalism 
below. Consider the parameter state label set corresponding to all parameters of type $X_{i}$. Let $\mathscr{X}$ be a single subset of the parameter state label set (called a "single region") and let $\mathscr{X}^{c}$ (the complement) be the set containing the remaining elements. Define the proportional change

$$
\begin{aligned}
& X_{i}(r)=r X_{i}, \quad i \in \mathscr{X}, \\
& X_{i}(r)=X_{i}, \quad i \in \mathscr{X}^{c}, \\
& Z_{j}(r)=Z_{j} .
\end{aligned}
$$

Hence a subset of the state parameters is scaled by a common factor $r$ (e.g., all pipe lengths can be scaled by a common factor, while other parameters are not scaled).

Most of the mathematical formalism that follows before Section 3.3 was previously developed as a first orientation [21], restricting to a single region and proportional change. The current work is comprehensive, due to including additive change and extending to multiple regions.

Equation (3) implies that the real number $r$ is mapped into the solution vector

$$
(\mathbf{q}(r), \mathbf{p}(r))=f(\mathbf{X}(r), \mathbf{Z}(r))
$$

Thus as $r$ varies, a line is traced out in solution space. Consider a function $g(\mathbf{q}, \mathbf{p})$, mapping into a real number. Define an indicator function $\xi$ as

$$
\xi: \Re \longmapsto \Re \quad \text { where } \xi(r) \equiv g(f(\mathbf{X}(r), \mathbf{Z}(r)))
$$

The inversion of $f$, which maps a vector into a vector, is often required for the full inverse solution of sensitivity problems. However, these problems will be studied here as an inversion of the much simpler function $\xi$, which maps a number into a number.

In this work a meaningful choice of $g$, relevant to model calibration [4, Section 14.5] [8], will be studied as an example. Define the specific instance of $g$ as $g_{a}$ and the specific instance of $\xi$ as $\langle p\rangle$, such that

$$
\begin{aligned}
g_{a}(\mathbf{q}, \mathbf{p}) & \equiv \frac{1}{N\left(\mathscr{M}^{\odot}\right)} \sum_{m \in \mathscr{M}^{\odot}} p_{m} \\
\langle p\rangle(r) & =\frac{1}{N\left(\mathscr{M}^{\odot}\right)} \sum_{m \in \mathscr{M}^{\odot}} p_{m}(\mathbf{X}(r), \mathbf{Z}(r))
\end{aligned}
$$

$g_{a}$ is the average pressure head in the internal nodes. $N\left(\mathscr{M}^{\odot}\right)$ is the number of labels in the set $\mathscr{M}^{\odot}$, i.e., the number of internal nodes.

3.2. Linearization: Proportional Change. In the case where some state parameters vary around the base-case parameters with $r$ very near to 1 , it follows that $\xi(r)$ is very near to $\xi(1)$. Particularly,

$$
\begin{aligned}
\Delta r & \equiv r-1, \\
|\Delta r| & \ll 1,
\end{aligned}
$$

$$
\begin{aligned}
\Delta \xi & \equiv \xi(r)-\xi(1)=\widehat{D} \Delta r, \\
\widehat{D} & \equiv \xi^{\prime}(1),
\end{aligned}
$$

(deriv. w.r.t. $r$ at $r=1$ ),

where the Taylor expansion is utilised. This equation can be used as follows in practical applications. Choose state parameters satisfying (3) with $r$ very near to 1 . Calculate $\Delta \xi$ using software, e.g., EPANET 2. Then use (7) to infer $\widehat{D}$.

Take the specified uncertainty for $\xi(r)$ to be

$$
\Delta \xi \quad \Delta \xi \text { positive or negative. }
$$

The deduced uncertainty is (from (7))

$$
\Delta r=\frac{\Delta \xi}{\widehat{D}}
$$

The procedure for a linear sensitivity study is hence suggested to be as follows. Obtain $\widehat{D}$ for a set of scaling state parameters $\mathscr{X}$, representing deviations around the base-case parameters. Then use (9) to obtain $\Delta r$ for a given value of $\Delta \xi$. The corresponding value of $r$ then yields the state parameter uncertainty limits by using (3).

In order for the procedure to be meaningful, $\Delta \xi$ must represent a tolerance that is allowable for the WDS. The sensitivity examples in this paper take the average pressure to have a certain tolerance. They therefore use $\xi=\langle p\rangle$ from (6).

Equation (9) is the main result used for the linear approximation. It is only exact for $r$ very near to 1 .

3.3. Parameter Variation: Additive Change. Consider the parameter state label set corresponding to all parameters of type $Z_{j}$. Let $\mathscr{Z}$ be a single subset of the parameter state label set and let $\mathscr{Z}^{c}$ (the complement) be the set containing the remaining elements. Define the additive change

$$
\begin{aligned}
& X_{i}(z)=X_{i}, \\
& Z_{j}(z)=Z_{j}+z, \quad j \in \mathscr{Z}, \\
& Z_{j}(z)=Z_{j}, \quad j \in \mathscr{Z}^{c} .
\end{aligned}
$$

Hence a subset of the state parameters (elevations) is increased by a common factor $z$.

Compare the additive change in (10) with the proportional change in (3). The analysis for additive change is very similar to that of proportional change, and (4)-(6) are obtained with all references to $r$ replaced with $z$, and $\xi(1) \rightarrow$ $\xi(0)$.

3.4. Linearization: Additive Change. The analogue to (7), for state parameters (elevations) varying around the reference parameters with $z$ very near to 0 , is

$$
\Delta z \equiv z,
$$

$|\Delta z| \ll 1$, 


$$
\begin{aligned}
\Delta \xi & \equiv \xi(z)-\xi(0)=\widetilde{D} \Delta z, \\
\widetilde{D} & \equiv \xi^{\prime}(0),
\end{aligned}
$$

(deriv. w.r.t. $z$ at $z=0$ ).

The analogue of (9) is

$$
\Delta z=\frac{\Delta \xi}{\widetilde{D}}
$$

which is only exact for $z$ very near to 0 . This equation gives the limits of the state parameters (elevations) according to (10). The procedure for a linear sensitivity study is as follows. Obtain $\widetilde{D}$ for a set of changing state parameters $\mathscr{Z}$, representing deviations around the base-case parameters. Then, given the value $\Delta \xi$, determine $\Delta z$ in accordance with (12).

3.5. Generalization of $g$. The specific instance of $g$ in (6) is defined in terms of the pressures at all the nodes. More generally, $g$ can be considered to be defined in terms of a subset $\mathscr{M}^{\oplus}$ of the internal nodes $m$ (at which pressures are calculated, and state parameters [elevations] are specified) and a subset $\mathscr{L}^{\oplus}$ of the links $l$ (at which flow rates are calculated, and state parameters are specified). Hence, as described in Section 2, there is the association in (1).

3.6. Time Dependence and Tank Diameter Scaling Law. Most of the quantities discussed here are in principle functions of time. For example, demand, flow rate, and pressure are usually functions of time. Although the formalism and the results of this paper apply to both steady-state and extended-period simulations, time dependence is not explicitly indicated.

The proportional or additive change is considered at a specific instant in time (and can be time-dependent) and is hence naturally suited to a state-state simulation. For an extended-period simulation, it is often meaningful to consider proportional or additive change that is independent of time.

Tank water surface level at the next time step $t+\Delta t$ is derived from the flow in or out of the tank at a specific instant in time $t[14]$ and is hence a "derived" quantity. It can be shown that the flow in or out of the tank at $t$ is not dependent on the diameter of the tank $D_{T}$, or the shape of the tank, but only on the water surface elevation in the tank [14]. Hence, we have the following.

Result 1 (tank diameter scaling). When only tank diameters are scaled, the change in $\xi$ can be calculated analytically by noting that the flow rates and pressures remain unchanged. If $\xi$ is not explicitly dependent on tank diameters, it will remain unchanged, implying that derivatives of $\xi$ for tank diameter scaling will be zero.

The latter statement implies that $\widehat{D}_{D_{T}}=0$. On the other hand, the tank water surface level at the next time step $t+\Delta t$ will depend on $D_{T}$, or the shape of the tank, unless there is no flow from the tank at time $t$ (i.e., it is in equilibrium or has reached the minimum or maximum level).

\section{Introduction to the Results: Single State Parameter Region}

It has been emphasized that uncertainty in pipe length, diameter, and roughness coefficient, as well as reservoir/tank surface elevation or head, needs to be taken into account in WDS analysis [18]. This will now be considered.

None of the results in this paper depend on the linearization approximation. However, the practical consequences of pipe property scaling use this approximation (around (16) and (23)).

Although the formalism in Sections 3.1 and 3.2 allows for a proportional change to apply to different types of parameters (e.g., lengths and diameters being scaled together), this possibility will not be explored further in this paper. Proportional changes will be studied for different types of parameters referring to a common set of links $l$ in $\mathscr{L}$. For example, scaling of all pipe lengths $L_{l}$ by $r_{L}$ will be studied, similarly for pipe diameters and roughness coefficients corresponding to the same set of links.

When additive change is studied, the following labels will refer to an elevation:

(i) $j$ : an internal or external node (as in (10));

(ii) $m$ : only an internal node;

(iii) $\mu$ : only an external node.

When results are stated without proof, the proof is in the Appendix. Sections 5 and 6 contain results for the case where there is a single state parameter region.

\section{Pipe Parameter Scaling Laws}

Assume that the major friction loss in a pipe is described by either the Hazen-Williams (H-W) or Chezy-Manning (C-M) formulae (defined in the Appendix), with $C$ and $N$ denoting the respective roughness coefficients. Scale the pipe length, diameter, and roughness coefficient by, respectively, $r_{L}, r_{D}$ and $r_{C}$ or $r_{N}$ for all links $\mathscr{L}$. Assume a general indicator function $\xi$ defined, as discussed in Section 3.5, in terms of flow rates, pressures, and state parameters.

Result 2 (pipe parameter scaling without minor losses). Assume that there are no minor losses in the pipes corresponding to the links $\mathscr{L}$. Assume that $\xi$ does not explicitly depend on any pipe length, diameter, or roughness coefficient for a link in $\mathscr{L}$. Then $\xi\left(r_{L}\right)=\xi\left(r_{D}\right)=\left[\xi\left(r_{C}\right)\right.$ or $\left.\xi\left(r_{N}\right)\right]$ if the parameter uncertainties are related by

$$
\begin{aligned}
\mathrm{H}-\mathrm{W}: \frac{1}{r_{D}^{4.871}} & =r_{L}=\frac{1}{r_{C}^{1.852}} \\
& \Downarrow \\
\widehat{D}_{D} & =-4.871 \widehat{D}_{L} \\
\widehat{D}_{C} & =-1.852 \widehat{D}_{L}, \\
\mathrm{C}-\mathrm{M}: \frac{1}{r_{D}^{16 / 3}} & =r_{L}=r_{N}^{2}
\end{aligned}
$$




$$
\begin{gathered}
\Downarrow \\
\widehat{D}_{D}=-\frac{16}{3} \widehat{D}_{L} \\
\widehat{D}_{N}=2 \widehat{D}_{L} .
\end{gathered}
$$

Result 3 (pipe parameter scaling with minor losses). Assume that there are minor losses in the pipes corresponding to the links $\mathscr{L}$. Assume that $\xi$ does not explicitly depend on any pipe length or roughness coefficient for a link in $\mathscr{L}$. Then $\xi\left(r_{L}\right)=$ $\left[\xi\left(r_{C}\right)\right.$ or $\left.\xi\left(r_{N}\right)\right]$ if the parameter uncertainties are related by

$$
\begin{aligned}
\text { H-W: } r_{L} & =\frac{1}{r_{C}^{1.852}} \Longrightarrow \\
\widehat{D}_{C} & =-1.852 \widehat{D}_{L}, \\
\text { C-M: } r_{L} & =r_{N}^{2} \Longrightarrow \\
\widehat{D}_{N} & =2 \widehat{D}_{L} .
\end{aligned}
$$

There are no corresponding results for the DarcyWeisbach (D-W) major friction formula. The Results reference the pipe length, because its dependence is the same for the H-W and C-M major friction loss formulae (see (A.1)), as well as for D-W [14]. The derivatives for the pipe length, diameter, and roughness coefficient scaling are denoted by $\widehat{D}_{L}, \widehat{D}_{D}$ and $\widehat{D}_{C}$ or $\widehat{D}_{N}$, respectively.

Examples of the applicability of the Results are as follows:

(i) If $\xi$ is only dependent on pressure (e.g., $\langle p\rangle$ ), flow rate, or piezometric head $H$ (which is dependent on pressure and elevation [14]), both Results may hold.

(ii) If $\xi$ is dependent on velocity in a pipe for a link in $\mathscr{L}$ (which is dependent on flow rate and pipe diameter according to (A.1)), only the second Result may hold.

(iii) For tank water surface elevations, which are commonly used for model calibration [4, Section 14.5] [8], both Results may hold (see the Appendix).

For a very small tolerance $\Delta \xi$, the change $\Delta r$ is very small, and the relationships between the $r$ 's in (13)-(14) in the first Result become, from the Taylor expansion,

$$
\begin{gathered}
\text { H-W: }-4.871 \Delta r_{D}=\Delta r_{L}=-1.852 \Delta r_{C}, \\
\text { C-M: }-\frac{16}{3} \Delta r_{D}=\Delta r_{L}=2 \Delta r_{N} .
\end{gathered}
$$

The relationships between the $r$ 's in (15)-(16) of the second Result are the same, with reference to $\Delta r_{D}$ removed in (16).

Assume the indicator function is known with a very small tolerance $\Delta \xi$, and an uncertainty in the pipe lengths is consistent with this tolerance (this can be calculated for the WDS for a single state parameter region via software from (9)). Then, assuming the assumptions stated in the Results above, the Results have the following practical implications:

(i) For Result 2: a pipe diameter uncertainty of -4.871 times smaller, or a roughness coefficient uncertainty of -1.852 times smaller than the pipe length uncertainty, is consistent with the tolerance $(\mathrm{H}-\mathrm{W})$. For C$\mathrm{M}$, the same statement holds with the replacement $-4.871 \rightarrow-16 / 3$ and $-1.852 \rightarrow 2$.

(ii) For Result 3: a pipe roughness coefficient uncertainty of -1.852 times smaller than the pipe length uncertainty is consistent with the tolerance (H-W). For C$\mathrm{M}$, the same statement holds with the replacement $-1.852 \rightarrow 2$.

There was a computer-based NRSA study of pipe length, diameter, and roughness variation for an Alpine WDS [10]. Specifically, uncertainly in roughness is especially frequently studied $[8,15,16,18]$.

\section{Analytical Calculation of Node Elevation Uncertainty}

The effect of uncertainty in the elevation of nodes that do not correspond to reservoirs or tanks (internal nodes) can be determined via a simple analytical calculation, using the following Result.

Result 4 (internal node elevation uncertainty). Consider internal nodes $m$ in $\mathscr{M}$. An additive change of elevation for the nodes $\mathscr{M}$ according to (10) means that all flow rates remain unchanged, and all state parameters and pressures remain unchanged, except the following:

$$
\begin{aligned}
& Z_{m} \longrightarrow Z_{m}+z \\
& \quad p_{m} \longrightarrow p_{m}-z \\
& \quad m \in \mathscr{M}
\end{aligned}
$$

The Result only applies if there are no pressure-dependent components in the WDS.

Note that an emitter, Pressure Reducing Valve, Pressure Sustaining Valve, Pressure Breaker Valve, or pressuredependent demand is a pressure-dependent component [5].

Let $\mathscr{M}^{\prime}$ denote the set of all external node labels $\mu$. It corresponds to the following state parameters:

(i) Reservoir water surface elevation (if the reservoir is under atmospheric pressure)

(ii) Reservoir piezometric head (if the reservoir is under pressure)

(iii) Tank water surface elevation

(iv) Tank bottom and top water surface elevation

The effect of uncertainty in the elevation of nodes that correspond to reservoirs or tanks (external nodes) can be determined via a simple analytical calculation, using the following Result.

Result 5 (external node elevation uncertainty). Consider an additive change for all state parameters corresponding to $\mathscr{M}^{\prime}$. 
A change according to (10) means that all flow rates and state parameters remain unchanged, except the following:

$$
\begin{array}{ll}
Z_{\mu} \longrightarrow Z_{\mu}+z^{\prime} & \mu \in \mathscr{M}^{\prime} \\
p_{m} \longrightarrow p_{m}+z^{\prime} & m \in \mathscr{M}^{\odot}
\end{array}
$$

The Result only applies if there are no pressure-dependent components in the WDS.

Examples of applications of the Results in this section to average pressure and tank water surface elevation are given in the Appendix.

A particularly practical application of Result 5 is obtained when the interest is in uncertainty due to reservoir water surface elevation (if the reservoirs are under atmospheric pressure). This can be the same for all reservoirs due to a common water level measurement uncertainty, or when rain or evaporation causes the water levels to change by the same amount. If the tank level evolution is such that the tank water surface elevation never goes below the minimum or maximum for the tank, either before or after the change, the tank bottom and top water surface elevations do not have to be changed (since the water level is then the only parameter on which the WDS depends). This means that Result 5 applies for the same change in reservoir water levels, as long as all tank water levels are changed by the same amount, which is a sensible possibility of considering. Hence only water levels are changed.

\section{Introduction to Results: Multiple State Parameter Regions}

So far, a common change for a single set of state parameters (corresponding to the region $\mathscr{X}$ or $\mathscr{Z}$ ) was considered. The effect of the change on a single indicator function was determined. Consider a generalization to multiple regions as follows.

Consider the parameter state label set corresponding to all parameters of type $X_{i}$. Let $\mathscr{X}_{k_{1}}$ be disjoint subsets of the parameter state label set and let $\mathscr{X}^{c}$ (the complement) be the set containing the remaining elements. Consider the parameter state label set corresponding to all parameters of type $Z_{j}$. Let $\mathscr{Z}_{k_{2}}$ be disjoint subsets of the parameter state label set and let $\mathscr{Z}^{c}$ be the set containing the remaining elements. Define the change

$$
\begin{aligned}
& X_{i} \longrightarrow r_{k_{1}} X_{i} \quad i \in \mathscr{X}_{k_{1}} \\
& X_{i} \longrightarrow X_{i} \quad i \in \mathscr{X}^{c} \\
& Z_{j} \longrightarrow Z_{j}+z_{k_{2}} \quad j \in \mathscr{Z}_{k_{2}} \\
& Z_{j} \longrightarrow Z_{j} \quad j \in \mathscr{Z}^{c}
\end{aligned}
$$

which describes a common proportional change for each of the regions $\mathscr{X}_{k_{1}}$ (compare (3)) and a common additive change for each of the regions $\mathscr{Z}_{k_{2}}$ (compare (10)). An example of a regional application of proportional change of $\mathrm{H}-\mathrm{W}$ roughness coefficients can be found in a numerical Monte Carlo and FOSM study (see Table 1 of [16]).
Generalizing to multiple indicator functions will allow inverse sensitivity analysis via studying the LRSA analogue of the sensitivity-matrix. In the case where each $\mathscr{X}_{k_{1}}$ and $\mathscr{Z}_{k_{2}}$ above refers to a single state parameter, the linearized version of the formalism would just be the sensitivity-matrix method. Although sensitivity-matrix methods are computationally efficient [9], they require a high cost of human effort and time and present difficulty in mathematical formulation and computer program implementation [3]. Therefore, full sensitivitymatrix methods will not be developed further here. Only a single indicator function will be considered, implying that results will only be useful for forward sensitivity analysis.

The results for multiple state parameter regions are generalizations of those for a single state parameter region and are hence stated without proof. Result 1 is identical to what was stated before but is now applied to scaling of multiple groups of tank diameters. Sections 8 and 9 contain further generalized results.

\section{Pipe Parameter Scaling Laws}

Scale the pipe length, diameter, and roughness coefficient as three multiple region scaling cases, where the regions are the same in each case:

$$
\begin{array}{rl}
\text { Length: } L_{l} \longrightarrow r_{L k} L_{l} & l \in \mathscr{L}_{k} \\
\text { Diameter: } D_{l} \longrightarrow r_{D k} D_{l} & l \in \mathscr{L}_{k} \\
\text { Roughness: H-W: } C_{l} \longrightarrow r_{C k} C_{l} & l \in \mathscr{L}_{k} \\
\text { C-M: } N_{l} \longrightarrow r_{N k} N_{l} & l \in \mathscr{L}_{k} .
\end{array}
$$

Let $\cup \mathscr{L}$ denote the union of the links in $\mathscr{L}_{k}$, i.e., all the links which correspond to pipe parameters that scale. Let $\left\{r_{k}\right\}$ denote all the scaling factors $r_{1}, r_{2}, \ldots, r_{k}, \ldots$

Result 6 (pipe parameter scaling without minor losses). Assume that there are no minor losses in the pipes corresponding to the links $\cup \mathscr{L}$. Assume that $\xi$ does not explicitly depend on any pipe length, diameter, or roughness coefficient for a link in $\cup \mathscr{L}$. Then $\xi\left(\left\{r_{L k}\right\}\right)=\xi\left(\left\{r_{D k}\right\}\right)=$ $\left[\xi\left(\left\{r_{C k}\right\}\right)\right.$ or $\left.\xi\left(\left\{r_{N k}\right\}\right)\right]$ (for the three cases in (20)) if the parameter uncertainties are related, for each region $k$, by

$$
\begin{gathered}
\mathrm{H}-\mathrm{W}: \frac{1}{r_{D k}^{4.871}}=r_{L k}=\frac{1}{r_{C k}^{1.852}}, \\
\mathrm{C}-\mathrm{M}: \frac{1}{r_{D k}^{16 / 3}}=r_{L k}=r_{N k}^{2} .
\end{gathered}
$$

Result 7 (pipe parameter scaling with minor losses). Assume that there are minor losses in the pipes corresponding to the links $\cup \mathscr{L}$. Assume that $\xi$ does not explicitly depend on any pipe length or roughness coefficient for a link in $\cup \mathscr{L}$. Then $\xi\left(\left\{r_{L k}\right\}\right)=\left[\xi\left(\left\{r_{C k}\right\}\right)\right.$ or $\left.\xi\left(\left\{r_{N k}\right\}\right)\right]$ (for the "length" and "roughness" cases in (20)) if the parameter uncertainties are related, for each region $k$, by

$$
\begin{aligned}
& \mathrm{H}-\mathrm{W}: r_{L k}=\frac{1}{r_{C k}^{1.852}}, \\
& \mathrm{C}-\mathrm{M}: r_{L k}=r_{N k}^{2} .
\end{aligned}
$$


In the case where each $\mathscr{L}_{k}$ represents only one link and where $\cup \mathscr{L}$ is all the pipe links, i.e., where all pipe lengths corresponding to all links are scaled by a different amount for each link, the two Results give the most general relationship between the sensitivity of the indicator function to pipe lengths, diameters, and roughness coefficients.

For a very small tolerance $\Delta \xi$, the change $\Delta r$ is very small, and (21) of the first Result becomes, from the Taylor expansion,

$$
\begin{gathered}
\text { H-W: }-4.871 \Delta r_{D k}=\Delta r_{L k}=-1.852 \Delta r_{C k}, \\
\text { C-M: }-\frac{16}{3} \Delta r_{D k}=\Delta r_{L k}=2 \Delta r_{N k} .
\end{gathered}
$$

Equation (22) of the second Result is the same, with reference to $\Delta r_{D k}$ removed in (23). The practical implications of (23) are similar to what was discussed in Section 5.

The following practical example elucidates a possible application. Consider a WDS with three suburbs modelled with the H-W major friction formula. The model must be calibrated for two of the suburbs (denoted by $k=1,2$ ). WDS calibration data being used are a pressure and flow meter reading and a measured tank water surface elevation. A suitable combination of the three measurements is chosen to define an indicator function. Assume that the indicator function (calibration data) is known with a small tolerance of $5 \%$ and that the model predicts a value for the indicator function outside the measured range (a "calibration error"). Assume that a numerical calculation yields that for suburbs $k=1,2$ pipe length uncertainties of $\Delta r_{L k}=-10 \%,-20 \%$ are consistent with the tolerance. The pipe lengths are actually known to a higher accuracy in the model. Hence the calibration data do not constrain the lengths. Assume that an estimate yields that pipe roughness coefficients and diameters are known to $8 \%$.

Equation (23) implies that the roughness coefficient uncertainties for suburbs $k=1,2$ are $\Delta r_{C k}=-\Delta r_{L k} / 1.852=$ $5 \%, 11 \%$. Only for the first suburb this implies that the calibration data constrain the roughness coefficients tighter than they are known, so that there is a need to investigate whether the calibration error is due to inaccurate roughness coefficients. If only the second suburb is modelled to have no minor losses, (23) implies that the diameter uncertainty for suburb 2 is $\Delta r_{D 2}=-\Delta r_{L 2} / 4.871=4 \%$. For suburb 2 this implies that the calibration data constrain the diameters tighter than they are known, so that there is a need to investigate whether the calibration error is due to inaccurate diameters.

\section{Analytical Calculation of Node Elevation Uncertainty}

Apply an additive change in such a way that it is the same for all internal node elevations in a region $\mathscr{M}_{k}$ and is the same for all elevations (or heads) corresponding to external nodes $\mathscr{M}^{\prime}$ (defined in Section 6)

$$
\begin{gathered}
Z_{m} \longrightarrow Z_{m}+z_{k} \quad m \in \mathscr{M}_{k} \\
Z_{\mu} \longrightarrow Z_{\mu}+z^{\prime} \quad \mu \in \mathscr{M}^{\prime}
\end{gathered}
$$

Let $\cup \mathscr{M}$ denote the union of the internal nodes in $\mathscr{M}_{k}$, i.e., all the internal nodes which correspond to elevations that change.

Result 8 (node elevation uncertainty). Consider internal nodes $m$ in $\mathscr{M}_{k}$, for some region $k$. Let $\mathscr{M}^{\prime}$ refer to all the external nodes $\mu$. An additive change of elevations (or heads) according to (24) means that all flow rates remain unchanged, and all state parameters and pressures remain unchanged, except the following:

$$
\begin{aligned}
& Z_{m} \longrightarrow Z_{m}+z_{k} \\
& p_{m} \longrightarrow p_{m}+z^{\prime}-z_{k} \\
& m \in \mathscr{M}_{k} \\
& p_{m} \longrightarrow p_{m}+z^{\prime} \quad m \notin \cup \mathscr{M} \\
& Z_{\mu} \longrightarrow Z_{\mu}+z^{\prime} \quad \mu \in \mathscr{M}^{\prime}
\end{aligned}
$$

The Result only applies if there are no pressure-dependent components in the WDS.

A special application of this Result is obtained when $z^{\prime}=$ 0 , i.e., when there are no changes for reservoirs and tanks (external nodes). For this application, there is no need for the restriction that all external node elevations (and heads) should change in the same way. In the case where each $\mathscr{M}_{k}$ represents only one node, and where $\cup \mathscr{U}=\mathscr{M}^{\odot}$, i.e., where all elevations corresponding to all internal nodes are changed by a different amount for each node, and there are no changes for reservoirs and tanks, the Result gives the most general sensitivity of the indicator function to internal node elevations.

An example application is now given for the WDS in Figure 1, which is assumed to have no pressure-dependent components. The water surface elevations of the reservoir and tank are assumed to be well known. Assume the six left-most nodes are in an area where it was later determined that the elevation was $3 \mathrm{~m}$ lower than that represented in the original model $(k=1)$. Also, another node should have represented the top of a building, which is $2 \mathrm{~m}$ higher than the ground level represented in the original model $(k=2)$. Then Result 8 implies that in the revised model the pressure head will be $3 \mathrm{~m}$ higher at the six left-most nodes and $2 \mathrm{~m}$ lower at the node which represents the top of a building.

\section{Types of Sensitivity Analysis Covered by LRSA}

In local regional sensitivity analysis (LRSA) state parameters change in the same way for all parameters defining the region. Different categories of change can be distinguished, with corresponding Results that apply, as shown in Table 1. The changes considered in this paper (19) apply to all these categories. Categories C-E "interpolate" between the most complex (Categories A-B) and least complex (Category F) parameter change commonly considered. 
TABLE 1: Different types of sensitivity analysis ordered from most to least complex.

\begin{tabular}{|c|c|c|c|c|c|c|}
\hline & $\begin{array}{c}\text { Which state parameters } \\
\text { change? } \mathrm{p}=\text { parameter }(\mathrm{s}) \\
\mathrm{m}=\text { multiple, } \mathrm{s}=\text { single }\end{array}$ & Cor & Max & $\begin{array}{l}\text { Number of } \\
\text { modifications }\end{array}$ & Results that apply & $\mathrm{R}$ \\
\hline A & All $\mathrm{p}$ individually & No & 1 & All & None & $\mathrm{m}$ \\
\hline B & All p of a type individually & No & 1 & $N_{p}$ & 1,6 & \multirow{2}{*}{$\mathrm{m}$} \\
\hline $\mathrm{C}$ & $\mathrm{P}$ of a type in $\mathrm{m}$ regions & Yes & $>1$ & $>1$ & 7,8 & \\
\hline $\mathrm{D}$ & All $p$ of a type in a s region & Yes & $>1$ & 1 & 5 & $\mathrm{~s}$ \\
\hline $\mathrm{E}$ & $\mathrm{P}$ of a type in a s region & Yes & $>1$ & 1 & 1,2 & \multirow{2}{*}{$\mathrm{s}$} \\
\hline $\mathrm{F}$ & One p individually & No & 1 & 1 & 3,4 & \\
\hline
\end{tabular}

The first column labels the category. The column "Cor" indicates whether state parameters are correlated in a specific way. The column "Max" indicates the maximum number of parameters that change in the same way. The column "Number of modifications" indicates the number of different modifications that are made to parameters, e.g., if there are two parameter regions in which change is made, there are two modifications. $N_{p}$ refers to the number of parameters that exist of a specific type. The enclosed boxes under "Results that apply, R" can apply to multiple categories. The column "R" indicates the number of parameter regions.

TABLE 2: The types of parameters to which the various Results apply.

\begin{tabular}{lccc}
\hline Name & Parameter types that change & s Result & m Result \\
\hline Tank scaling & Diameter & 1 & 1 \\
Pipe scaling & Length, diameter, roughness coefficient & 2,3 & 6,7 \\
Elevation change & Any elevation & 4,5 & 8 \\
\hline
\end{tabular}

$\mathrm{s}=$ "single region" and $\mathrm{m}=$ "multiple region".

The formalism for change in a region corresponding to more than one parameter assumed that the parameter change in the region is correlated, in either a proportional (3) or additive (10) way. However, the specific choice of correlation is only a restriction when there is more than one parameter that changes in the same way, i.e., for Categories C-E. These categories are unique to the LRSA developed here. The study of Categories $\mathrm{F}$ and $\mathrm{A}-\mathrm{B}$ changes reduces to conventional methods, respectively, NRSA and (when linearized) the sensitivity-matrix.

As discussed in Section 1, sensitivity analysis can be used forward or in inverse. Although both directions of use can in principle be accommodated in the formalism developed here, only Categories D-F (single state parameter region) have been developed in both directions. Categories A-C (multiple regions) have only been developed for the forward direction, for the reasons explained in Section 7.

\section{Conclusions}

To study the sensitivity of a WDS with respect to parameters, the sensitivity of some property of the WDS needs to be measured. This property is quantified by the indicator function $\xi$. The types of parameters to which the various Results apply are summarized in Table 2.

The tank scaling Result states that flow rates and pressures obtained by solving the WDS equations at a specific instant in time are independent of tank diameters. Also, $\xi$ is usually completely independent of tank diameters, and if not, the dependence can be calculated analytically.

The pipe scaling Results give a relationship between pipe length and pipe roughness coefficient scaling, for two of the three commonly used major friction loss formulae. For most choices of $\xi$, the WDS is $1.852-2$ times more sensitive to roughness coefficients than to lengths. When certain pipes do not have minor losses, there is an additional relationship between pipe length and pipe diameter scaling. For most choices of $\xi$, the WDS is 4.871-5.333 times more sensitive to pipe diameters than to lengths. The WDS is hence the most sensitive to pipe diameters, medium sensitive to pipe roughness coefficients, and least sensitive to pipe lengths.

The elevation change Results apply to a WDS with no pressure-dependent components (e.g., sprinklers and certain valves) and have two main application areas:

(1) When all reservoir and tank elevations (and heads) remain the same, changes of other elevations do not change flow rates and change the pressures in a simple additive way.

(2) When all the reservoir water surface elevations are changed together, and the tanks do not reach the bottom or top of their allowed water surface elevation ranges, the flow rates remain unchanged, and the pressures change in a simple additive way.

The dependence of $\xi$ on elevation can be calculated analytically.

The Results about the sensitivity of the WDS, stated here, are general mathematical (specifically, analytical) results. The Results hence contribute to the theoretical understanding of the nature of a WDS. Because the Results are stated analytically, they can be used for practical engineering, without the need for specialised software. They also provide a cross-check for the veracity of computer-based sensitivity analyses.

The Results state the dependence of the WDS on state parameter regions for the first time. This is made possible by 
the novel sensitivity approach, based on regions, developed here.

Future work can apply the approach to breaking a large WDS into suburbs, in order to study a complicated problem incrementally. For example, model calibration can be performed by combining the calibration data to define a suitable $\xi$ for the WDS. Within a suburb, the dependence of $\xi$ (the calibration data) on various state parameter categories can then be determined numerically (for example, dependence on pipe roughness coefficients, water demands, pump parameters, and reservoir water surface elevations). This determines how accurately each category of state parameter needs to be determined within each suburb.

\section{Appendix}

\section{Single Region Proofs and Examples}

Section 5. The H-W and C-M major friction loss formulae and the minor loss formula (for SI units) are $[5,14]$

$$
\begin{aligned}
h_{f, \mathrm{HW}} & =\frac{10.68 L q^{1.852}}{C^{1.852} D^{4.871}} \\
h_{f, \mathrm{CM}} & =\frac{10.29 N^{2} L q^{2}}{D^{16 / 3}} \\
h_{f, M} & =K \frac{v^{2}}{2 g}=\frac{8 K q^{2}}{\pi^{2} g D^{4}}
\end{aligned}
$$

The H-W pipe head-loss for a pipe on link $l$ is, before the proportional change,

$$
h_{\mathrm{HW}}(1)=\frac{10.68 L_{l} q_{l}^{1.852}(1)}{C_{l}^{1.852} D_{l}^{4.871}}+\frac{8 K_{l} q_{l}^{2}(1)}{\pi^{2} g D_{l}^{4}},
$$

where "(1)" indicates the variable before the change. For a link in $\mathscr{L}$ the length scaling yields

$$
h_{\mathrm{HW}}\left(r_{L}\right)=r_{L} \frac{10.68 L_{l} q_{l}^{1.852}\left(r_{L}\right)}{C_{l}^{1.852} D_{l}^{4.871}}+\frac{8 K_{l} q_{l}^{2}\left(r_{L}\right)}{\pi^{2} g D_{l}^{4}} .
$$

A similar expression is obtained for roughness coefficient scaling. If the relationship between $r_{L}$ and $r_{C}$ in (15) holds, $h_{\mathrm{HW}}\left(r_{L}\right)=h_{\mathrm{HW}}\left(r_{C}\right)$ when $q_{l}\left(r_{L}\right)=q_{l}\left(r_{C}\right)$. This means that the same solution $\left(\mathbf{q}\left(r_{L}\right), \mathbf{p}\left(r_{L}\right)\right)=\left(\mathbf{q}\left(r_{C}\right), \mathbf{p}\left(r_{C}\right)\right)$ is produced by the two proportional changes (see (4)). This establishes Result 3 for the H-W case. The proof of the C-M case follows a similar argument.

Because the minor friction loss depends on $D_{l}$, the argument cannot be extended to pipe diameter scaling. However, for the case $K_{l}=0$ (no minor loss) this problem disappears, and Result 2 is obtained.

The relationships between $r_{L}, r_{D}$ and $r_{C}$ or $r_{N}$ in (13)-(15) imply the relationships between $\widehat{D}_{L}, \widehat{D}_{D}$ and $\widehat{D}_{C}$ or $\widehat{D}_{N}$ by use of the Taylor expansion and (7).

Example of Both Results. Tank water surface elevation: a proportional change in pipe length and roughness coefficient related by (15) (and in addition pipe diameter change, related by (13), if there are no minor losses for pipes corresponding to the links $\mathscr{L}$ ) gives the same time evolution of the tank water surface elevation.

Section 6. The Results follow for a WDS where the solution of the equations is fully determined by the flow rates $q_{l}$ for all links $l$ and piezometric heads $H_{j}$ for all internal and external nodes $j$. In such a WDS pressure head does not need to be specified to obtain a solution and is purely a derived quantity computed from $H_{m}$ via $p_{m}=H_{m}-Z_{m}$, for all internal nodes $m$. There are no pressure-dependent components which need the pressure to be specified to obtain the solution. The Results follow from the following observations. If $\left(q_{l}, H_{j}\right)$ is a solution before the change, then one has the following:

(i) For Result 4: $\left(q_{l}, H_{j}\right)$ is a solution after the change (this can be viewed as a special case of the next observation with $\left.z^{\prime}=0\right)$.

(ii) For Result 5: $\left(q_{l}, H_{j}+z^{\prime}\right)$ is a solution after the change, using the translational invariance of the entire WDS w.r.t. elevation, and hence head, change.

The Results then follow by noting that, for external nodes $\mu$, $H_{\mu}=Z_{\mu}$.

Example of Result 4. Average pressure: using $\langle p\rangle$ from (6) a simple analytical calculation shows that the change $Z_{m} \rightarrow$ $Z_{m}+z$ means that

$$
\begin{aligned}
\langle p\rangle(z) & =\langle p\rangle(0)-\frac{N(\mathscr{M})}{N\left(\mathscr{M}^{\odot}\right)} z \Longrightarrow \\
\widetilde{D} & =-\frac{N(\mathscr{M})}{N\left(\mathscr{M}^{\odot}\right)} .
\end{aligned}
$$

Example of Result 5. Average pressure: using $\langle p\rangle$ the change $Z_{\mu} \rightarrow Z_{\mu}+z^{\prime}$ means that

$$
\langle p\rangle(z)=\langle p\rangle(0)+z^{\prime} \Longrightarrow \widetilde{D}=1 .
$$

Example of Both Results. Tank water surface elevation: either the change $Z_{m} \rightarrow Z_{m}+z$ (in connection with Result 4) or the change $Z_{\mu} \rightarrow Z_{\mu}+z^{\prime}$ (in connection with Result 5) leaves the tank water surface elevation relative to the bottom and top elevations, as well as all flow rates, unchanged. Hence the time evolution of the tank water surface elevation relative to the bottom and top water surface elevations remains unchanged for both changes.

\section{Conflicts of Interest}

The author declares that there are no conflicts of interest regarding the publication of this paper.

\section{References}

[1] W. W.-G. Yeh, "Review of parameter identification procedures in groundwater hydrology: the inverse problem," Water Resources Research, vol. 22, no. 2, pp. 95-108, 1986.

[2] A. C. Cullen and H. C. Frey, Probabilistic Techniques in Exposure Assessment, Plenum Press, 1999. 
[3] H. C. Frey and S. R. Patil, "Identification and review of sensitivity analysis methods," Risk Analysis, vol. 22, no. 3, pp. 553-578, 2002.

[4] L. W. Mays, Water Distribution Systems Handbook, McGrawHill, 2000.

[5] L. A. Rossman, “EPANET 2 User Manual," Tech. Rep. EPA/600/ R-00/057, 2000.

[6] American Water Works Association, "Computer modeling of water distribution systems," in Manual of Water Supply Practices - M32, pp. 1-249, 3rd edition, 2012.

[7] J. Deuerlein, O. Piller, I. M. Arango, and M. Braun, "Parameterization of offline and online hydraulic simulation models," in Proceedings of the 13th Computer Control for Water Industry Conference, CCWI '15, vol. 119 of Procedia Engineering, pp. 545553, Elsevier Ltd., 2015.

[8] D. Sumer and K. Lansey, "Effect of uncertainty on water distribution system model design decisions," Journal of Water Resources Planning and Management, vol. 135, no. 1, pp. 38-47, 2009.

[9] J. Izquierdo, I. Montalvo, R. Pérez, and M. Herrera, "Sensitivity analysis to assess the relative importance of pipes in water distribution networks," Mathematical and Computer Modelling, vol. 48, no. 1-2, pp. 268-278, 2008.

[10] M. Möderl, C. Hellbach, R. Sitzenfrei et al., "GIS based applications of sensitivity analysis for water distribution models," in Proceedings of the World Environmental and Water Resources Congress 2011: Bearing Knowledge for Sustainability, E. R. Beighley and M. W. Killgore, Eds., American Society of Civil Engineers, pp. 129-136, 2011.

[11] R. Sitzenfrei, M. Mair, M. Möderl, and W. Rauch, "Cascade vulnerability for risk analysis of water infrastructure," Water Science and Technology, vol. 64, no. 9, pp. 1885-1891, 2011.

[12] G. Fu, Z. Kapelan, and P. Reed, "Reducing the complexity of multiobjective water distribution system optimization through global sensitivity analysis," Journal of Water Resources Planning and Management, vol. 138, no. 3, pp. 196-207, 2012.

[13] R. Sitzenfrei and W. Rauch, "Integrated hydraulic modelling of water supply and urban drainage networks for assessment of decentralized options," Water Science and Technology, vol. 70, no. 11, pp. 1817-1824, 2014.

[14] N. Trifunovic, Introduction to Urban Water Distribution, Unesco-IHE Lecture Note Series, Taylor and Francis Group, 2008.

[15] C. J. Hutton, Z. Kapelan, L. Vamvakeridou-Lyroudia, and D. A. Savić, "Dealing with uncertainty in water distribution system models: a framework for real-time modeling and data assimilation," Journal of Water Resources Planning and Management, vol. 140, no. 2, pp. 169-183, 2014.

[16] K. Lansey, "Uncertainty in water distribution network modeling," Journal of Contemporary Water Research and Education, vol. 103, no. 1, pp. 22-26, 1996.

[17] A. Bargiela and G. D. Hainsworth, "Pressure and flow uncertainty in water systems," Journal of Water Resources Planning and Management, vol. 115, no. 2, pp. 212-229, 1989.

[18] P. Sivakumar, R. K. Prasad, and S. Chandramouli, "Uncertainty analysis of looped water distribution networks using linked EPANET-GA method," Water Resources Management, vol. 30, no. 1, pp. 331-358, 2016.

[19] R. Pérez, V. Puig, J. Pascual, J. Quevedo, E. Landeros, and A. Peralta, "Methodology for leakage isolation using pressure sensitivity analysis in water distribution networks," Control Engineering Practice, vol. 19, no. 10, pp. 1157-1167, 2011.
[20] G. Sanz and R. Pérez, "Sensitivity analysis for sampling design and demand calibration in water distribution networks using the singular value decomposition," Journal of Water Resources Planning and Management, vol. 141, no. 10, Article ID 04015020, 2015.

[21] P. R. Page, "Smart optimisation and sensitivity analysis in water distribution systems," in Proceedings of the Smart and Sustainable Built Environments SASBE '15, J. Gibberd and D. C. U. Conradie, Eds., pp. 101-108, University of Pretoria, Pretoria, South Africa, 2015.

[22] S. Yoyo, P. R. Page, S. Zulu, and F. A'Bear, "Addressing water incidents by using pipe network models," in Proceedings of the WISA Biennial 2016 Conference and Exhibition, p. 130, WISA, Johannesburg, South Africa, 2016.

[23] M. S. Osman, A. M. Abu-Mahfouz, P. R. Page, and S. Yoyo, "Real-time dynamic hydraulic model for water distribution networks: steady state modelling," in Proceedings of the 6th IASTED International Conference: Environment and Water Resource Management (AfricaEWRM '16), pp. 142-147, ACTA Press, Calgary, Canada, 2016. 


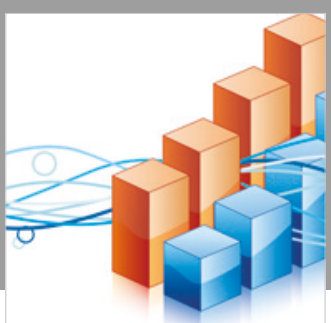

Advances in

Operations Research

\section{-n-m}
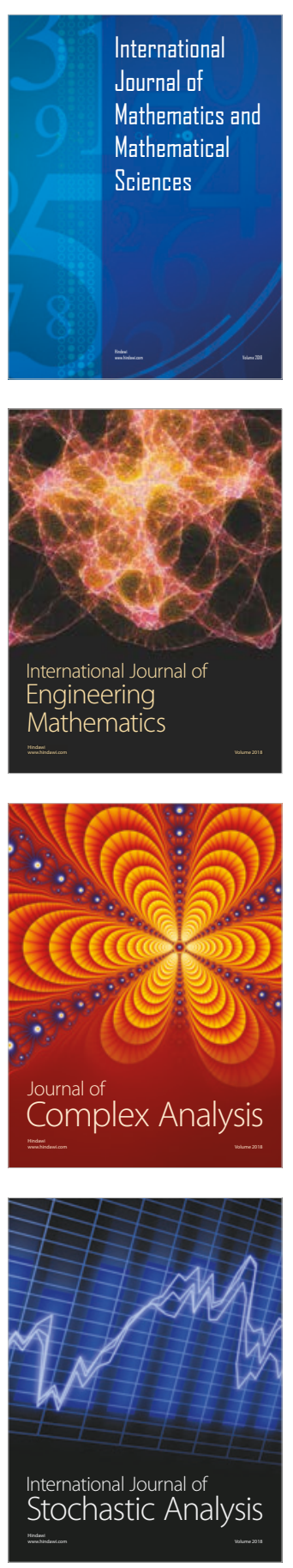
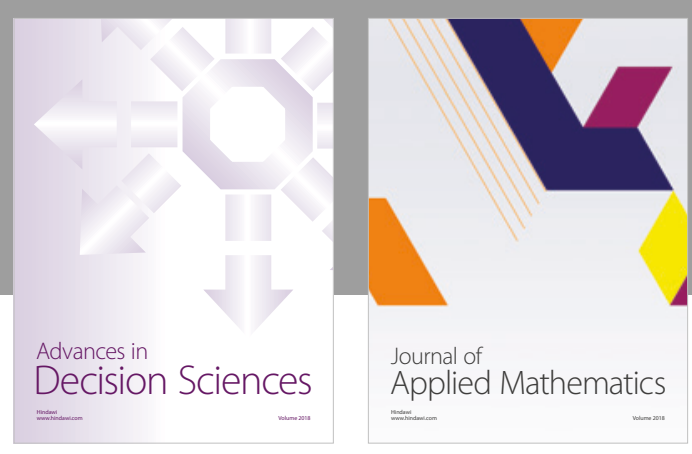

Journal of

Applied Mathematics
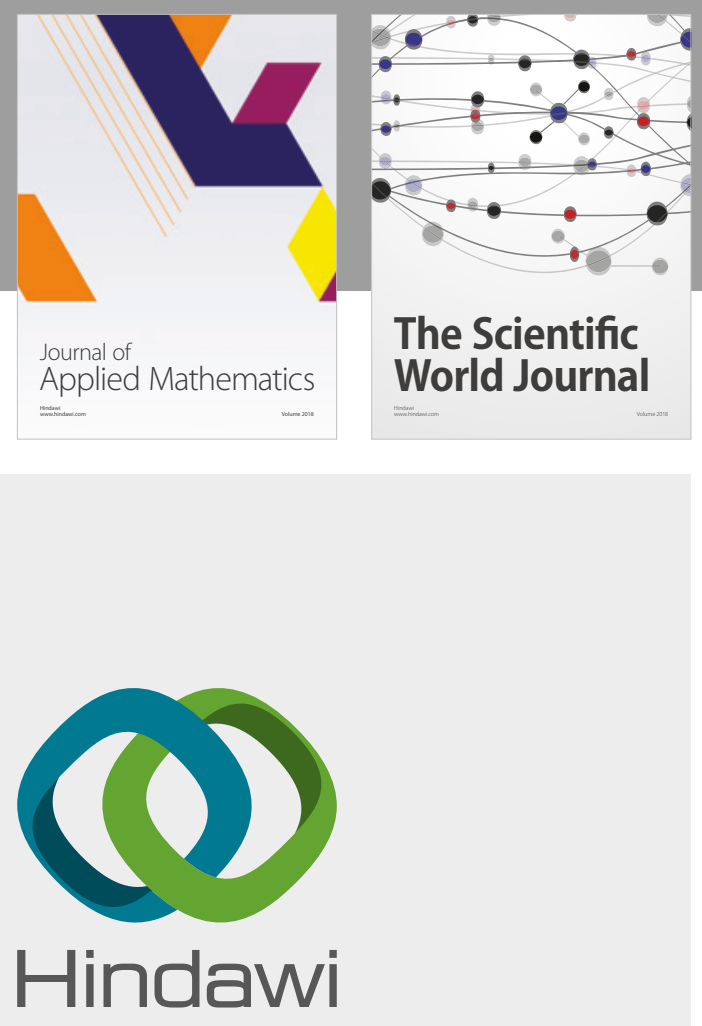

Submit your manuscripts at

www.hindawi.com

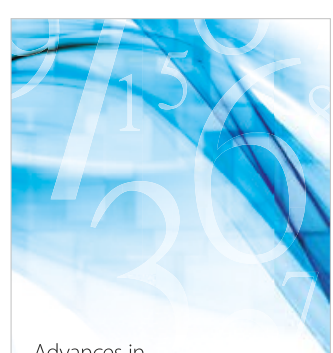

Advances in
Numerical Analysis
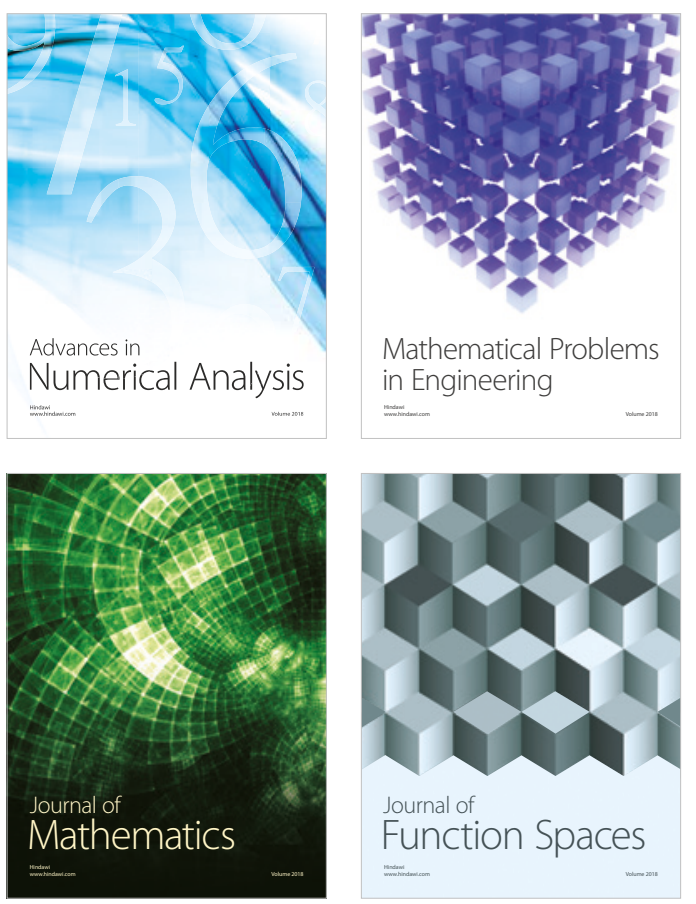

Mathematical Problems in Engineering

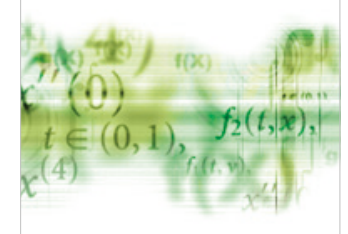

International Journal of

Differential Equations

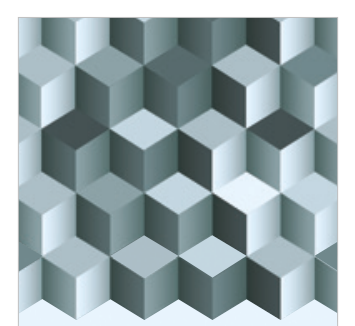

Journal of

Function Spaces

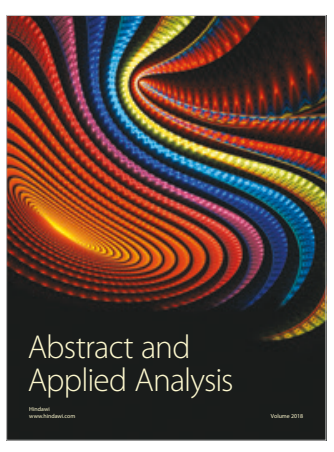

The Scientific

World Journal

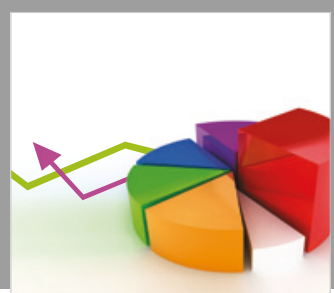

Journal of

Probability and Statistics
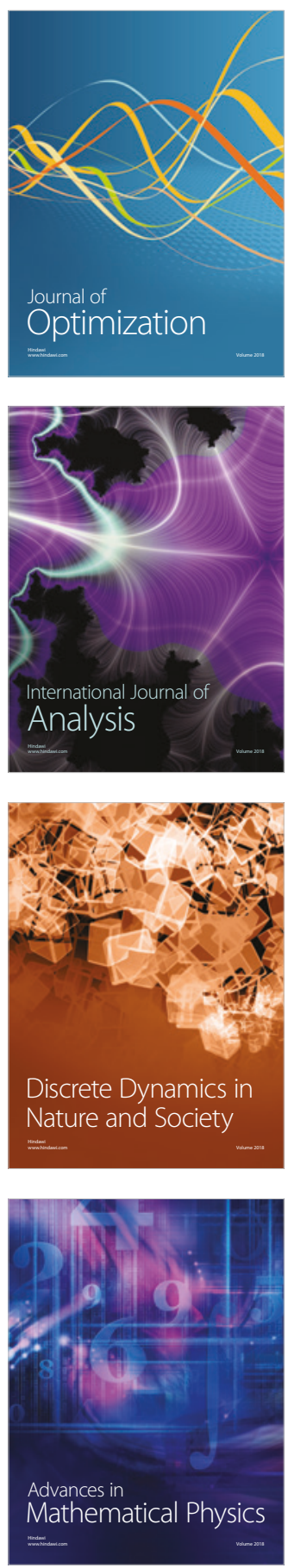